\title{
The Evolution and perturbation of Solitons in Dispersive- Nonlinear Optical Fiber
}

\author{
Cherinet Seboka Ambaye ${ }^{1, a}$,Guoping Zhang ${ }^{2}$, Yunhu $\mathrm{Wu}^{3}$ \\ 1,2,3 (College of Physical Science and Technology, Central China Normal University, Wuhan 430079,China) \\ a (Department of Physics, Madawalabu University, Bale Robe 217,Ethiopia
}

\begin{abstract}
A system of coupled nonlinear Schrodinger (NLSEs) of two fundamental and dark solitons are numerically investigated by Split-Step Fourier Method (SSFM) simulation. The stability of the evolved 'bright' envelopes as the superimposed of two dark solitons has been compared with the interaction of bright fundamental solitons in the perturbed or lossy fiber. Dynamic nature of the solitons in the the presence of nonlinear-dispersive phenomena, their relative amplitude $r_{i}$ relative phase $\theta_{i}$ and their separation distance
\end{abstract} $q_{0}\left(r_{0}\right)$ are evaluated in the simulation.

Keywords: Chirped-Gaussian Soliton, Dark and 'bright' Soliton, Solitons from Perturbation, Split-Step Fourier Method.

\section{Introduction}

Soliton based optical communication-systems have drawn wide attention in recent years because of their potential for increasing the capacity of the long-haul light wave systems. Optical solitons propagate in the form of light wave envelopes in optical fibers. The optical soliton is formed by a balance of the group velocity dispersion and the nonlinear change of the index of refraction Kerr effect of a fiber [1,2]. Although the nonlinear effect is extremely weak, its cumulative effect becomes significant since the communication distance measured by the light wavelength is extremely large. The dispersion phenomenon is a problem for high bit rate and long haul optical communication systems. Self-phase modulation (SPM) is the frequency change caused by a phase shift induced by the pulse itself. SPM arises because the refractive index of the fiber has an intensity dependent component $[3,4]$. When an optical pulse travels through the fiber, the higher intensity portions of an optical pulse encounter a higher refractive index of the fiber compared with the lower intensity portions.

This paper is able to demonstrate reshaping, appearance and symmetry distribution of solitons either from interaction or small perturbation in the dispersive nonlinear medium depending upon their relative amplitude $\mathrm{r}$, phase angle $\theta$ and their periodic separation q. The evolved bright envelope has been referred choice in transmitting optical data since the Gaussian envelope of fundamental solitons would spatially spread out infinitely in the fiber resulting in inter symbol interference (ISI) shown fig.1

\section{Modelling And Simulation}

Modelling and Simulation of solitons propagation is relied on solving the popular a generalized nonlinear Schrodinger Equation (NLSE) by Split-Step Fourier Method (SSFM) algorithm over lossless optical fiber[5, 6, 7]. The Nonlinear Schrodinger Equation (NLSE) is commonly written

$$
\frac{\partial A}{\partial z}+\frac{\partial \beta_{2}}{2} \frac{\partial^{2} A}{\partial T^{2}}-\frac{\beta_{3}}{6} \frac{\partial^{3} A}{\partial T^{3}}+\frac{\alpha}{2} A=i \gamma\left(|A|^{2} A+\frac{i}{\omega_{0}} \frac{\partial}{\partial T}(|A|)^{2} A-T_{R} A \frac{\partial|A|^{2}}{\partial T}\right)
$$

The differential operators for dispersion $\hat{D}$ and nonlinear $\hat{N}$ term are represented by

$$
\begin{aligned}
& \hat{D}=-\frac{\partial \beta_{2}}{2} \frac{\partial^{2}}{\partial T^{2}}+\frac{\beta_{3}}{6} \frac{\partial^{3}}{\partial T^{3}}-\frac{\alpha}{2} \\
& \hat{N}=i \gamma\left(|A|^{2}+\frac{i}{\omega_{0}} \frac{\partial}{\partial T}(|A|)^{2}-T_{R} A \frac{\partial|A|^{2}}{\partial T}\right)
\end{aligned}
$$

Treating the linear and nonlinear terms separately over step size $\mathrm{h}$ to administrate the simulation of split step Fourier (SSFM) algorithm is written as 


$$
A(T, z+h)=F T^{-1}\left[e^{\hat{D h}} F T\left[e^{\hat{N h}} A(T, z)\right]\right]
$$

The accuracy of the split step Fourier method can be improved, in a similar way to the midpoint as $[8,9]$

$$
\begin{aligned}
& A_{1}(T, z)=F T^{-1}\left[e^{\frac{\hat{D} h}{2}} F T\left[e^{\frac{\hat{N} h}{2}} A(T, z)\right]\right. \\
& A(T, z+h)=F T^{-1}\left[e^{\frac{\hat{D} h}{2}} F T\left[e^{\frac{\hat{N} h}{2}} A_{1}(T, z)\right]\right.
\end{aligned}
$$

\section{Discussion Results}

\subsection{Chirped Super-Gaussian Soliton in the presence of GVD and SPM}

The nonlinear Schrödinger equation NLSE can be modeled and simulated for lossless medium by suppressing or neglecting higher order dispersion and nonlinear parameters, then eq(1) becomes

$$
\frac{\partial A}{\partial z}+\frac{\partial \beta_{2}}{2} \frac{\partial^{2} A}{\partial T^{2}}-i \gamma\left(|A|^{2} A\right)=0
$$

Normalizing eq.(5) by introducing three dimensionless variables $\mu=\frac{A}{\sqrt{P_{0}}}, \xi=\frac{z}{L_{D}}, \tau=\frac{T}{T_{0}}$, where $P_{0}$ is the input optical power, $\mathrm{T}_{0}$ is temporal optical width, $L_{D}=\frac{T_{0}^{2}}{\left|\beta_{2}\right|}$, dispersion length, rewriting eq(5) as $i \frac{\partial \mu}{\partial \xi}-\operatorname{sgn}\left(\beta_{2}\right) \frac{1}{2} \frac{\partial^{2} \mu}{\partial \tau^{2}}+N^{2} \gamma|\mu|^{2} \mu=0$

Where $N^{2}=\frac{L_{D}}{L_{N L}}, L_{N L}=\frac{1}{\gamma P_{0}}$ is nonlinear length.

Assuming the chirped Gaussian pulse of $\mu=\exp \left(-0.5(1+i C) \tau^{2 m}\right)$ is the solution of eq(6) and launched and launched into lossless optical fiber. Where $\mathrm{m}$ is controlling the degree of edge sharpness, $\mathrm{C}$ is chirped parameter. The outcome of the simulation result based on split-step Fourier method algorithm is displayed on fig.1.

As it is observed on fig.1, the envelope of the soliton remains unaltered and undistorted as it launching in the presence of both GVD and SPM. A chirped pulse can be compressed during the early stage of propagation whenever the GVD parameter $\beta_{2}$ and the chirp parameter $\mathrm{C}$ happen to have $\beta_{2} C<0$. It is not difficult to imagine that under certain condition in such a way that SPM induced chirp of spectral broadening is just right to cancel the GVD compression of the pulse. 


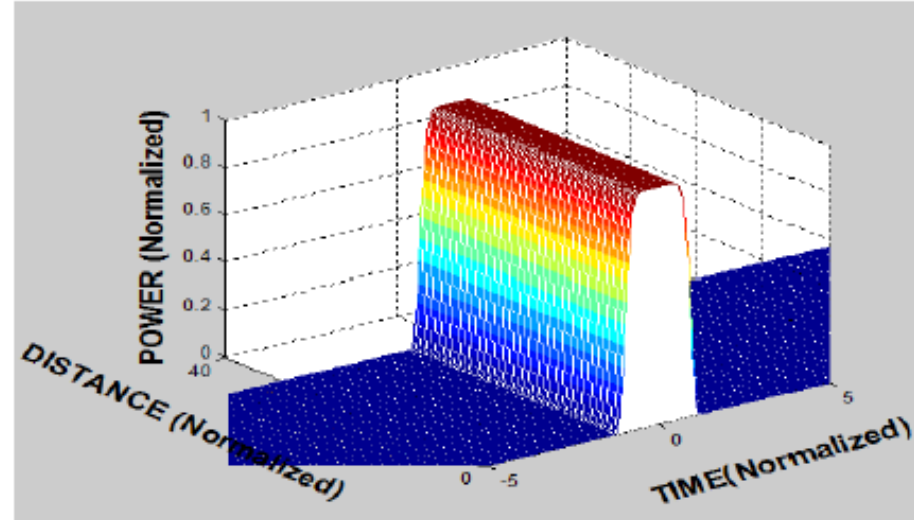

Figure 1: Undistorted soliton in the presence of both GVD and SPM.

\subsection{Soliton-Soliton Interaction in Optical Fiber}

Two soliton at the fiber input can be written as

$$
\mu(0, z)=\sec h\left(\tau-q_{0}\right)+r \operatorname{sech}\left[r\left(\tau+q_{0}\right)\right] e^{i \theta}
$$

where $r$ is the a parameter that determines a relative amplitude and $\theta$ is a relative phase between the solitons. The capacity of bit rates B that the solitons hold is given by eq(8). $q_{0}$ is the minimum distance, (experimentally $q_{0}=3: 5$ ) between the solitons. The bit rate $\mathrm{B}$ of the solitons in the system is described as[10, 11]:

$$
B=\frac{1}{2 q_{0} \tau_{0}}
$$

The interaction between the solitons depends not only on their separation distance but on their relative has and amplitude as well. If both pulses have nearly the same amplitude, their motion can be described by interaction force which exponentially decreases with distance between them and varies sinusoidally with their relative phase. The distance between the solitons is equal to $\mathrm{q}$ at every point in the waveguide (optical fiber) [11, $13,17]$ as

$$
q(\xi)=q_{0}+\ln \left|\cos \left(2 \xi e^{-q_{0}}\right)\right|
$$

The period of equation becomes $q(\xi)=q_{0}$ after a distance

$$
\xi_{p}=\frac{1}{2} e^{q_{0}} \cos ^{-}\left(e^{-q_{0}}\right) \approx \frac{\pi}{4} e^{q_{0}}
$$

A possibility for the suppression of the interaction is to choose a value for much longer than the fiber length. This can be done by a wider distance between the solitons at the fiber input but the result is a reduction of the bit rate in the system. The real collision length $L_{c o l}$ in the fiber between collisions of the solitons is[11]

$$
L_{c o l}=\frac{\pi}{2} L_{D} \exp \left(q_{0}\right)
$$

From fig.2, the minimum allowable separation distance is $q_{0}=3.5$ it has been observed that there is no overlap between the two solitons. The transmission data rate would transfer safely without deterioration. 


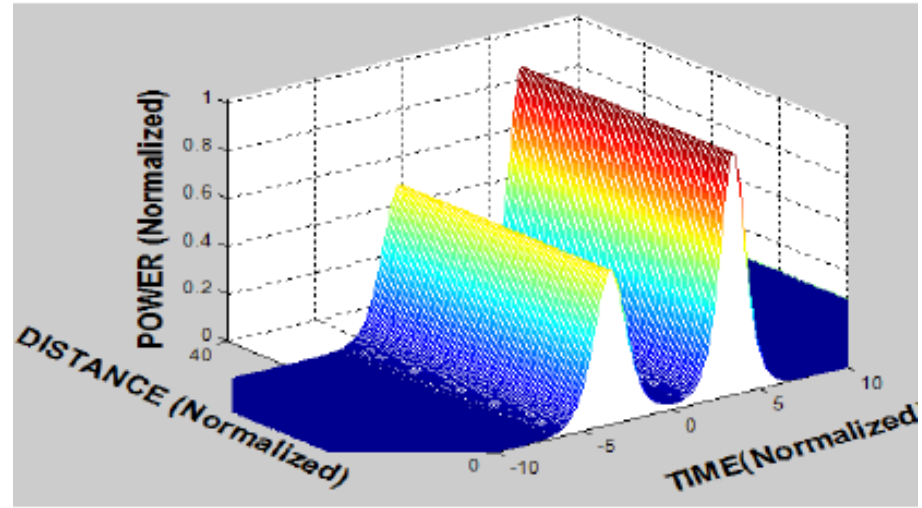

Figure 2: Evolution of solitons whose relative amplitude at $r=0.8$, The separation distance is $\mathrm{q} 0=3.5$, the relative phase $\theta=0$.

From fig.3, the separation distance becomes $\mathrm{q} 0=7.0$ and their relative phase is $\frac{3 \pi}{2}$.The transmission rate is even more relaxed than the phenomena observed in fig. 2 at the expense of decreasing the bit rate according to $\mathrm{eq}(8)$.

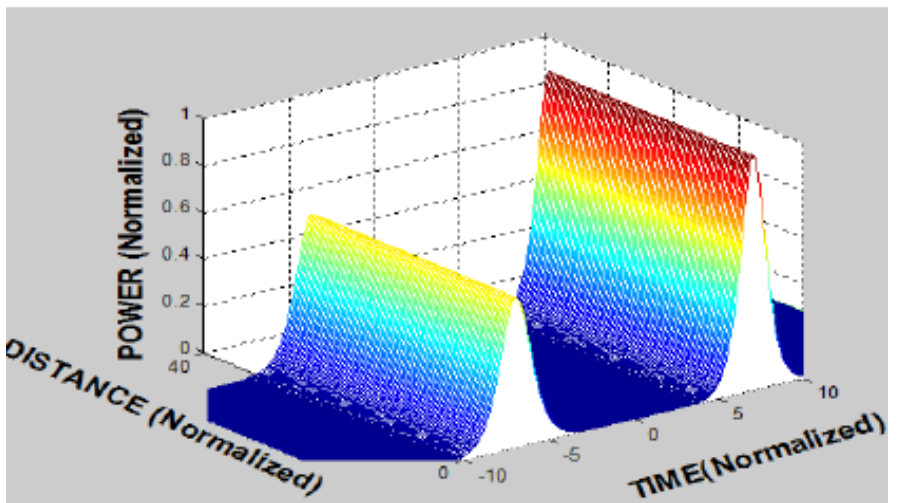

Figure 3: Evolution of solitons whose relative amplitude at $\mathrm{r}=0.8$, the separation distance $\mathrm{q} 0=7.0$, the relative phase $\theta=3 \pi / 2$

When the separation distance is reduced below the allowable magnitude, the interaction of the soliton could increase dramatically as it is observed on fig.4.This phenomena brings the deterioration of the transmission data rate as occurrence of inter symbol interference(ISI).

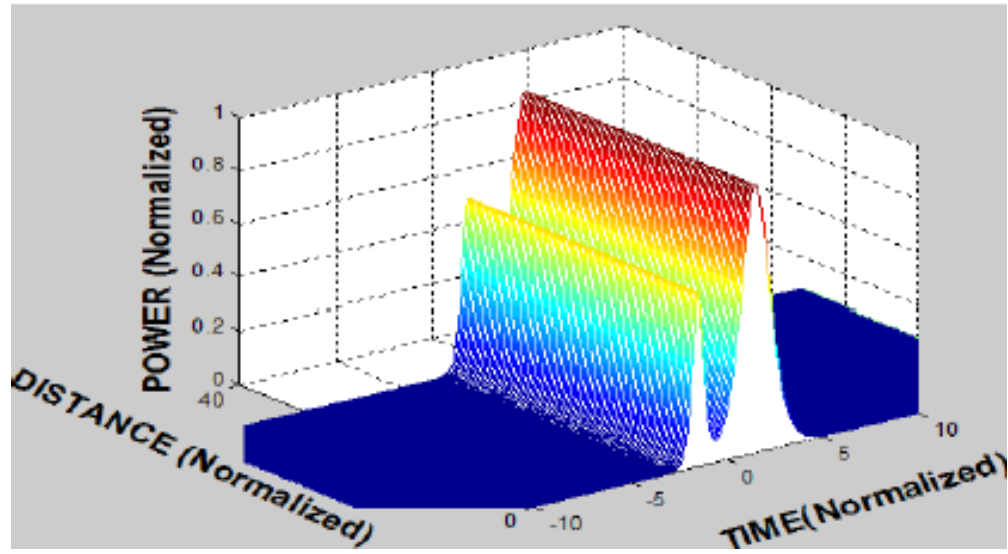

Figure 3: Evolution of solitons whose relative amplitude at $r=0.8$, the separation distance $q 0=1.5$ the relative phase $\theta=3 \pi / 2$ 
As seen on fig.5, the relative amplitude of the solitons are let to be increased from 0.8 to 2.5 with distance separation $\mathrm{q} 0=3: 5$ and relative phase angle $\theta=\frac{3 \pi}{2}$, there is a transfer of power from one soliton to the other, so that one soliton gradually disappears in long transmission distance. This phenomenon undermines the ideal transmission using solitons in long haul optical fiber communication.

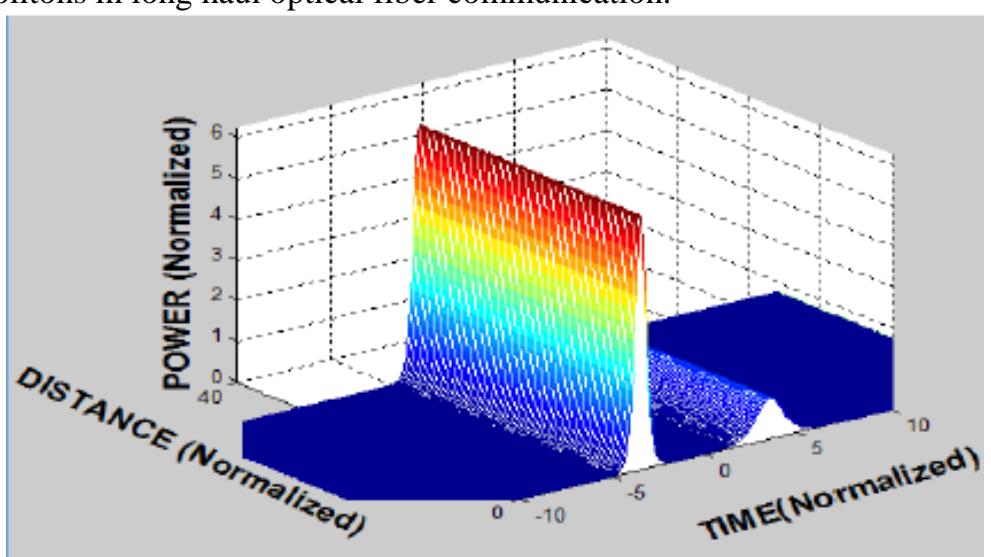

Figure 3: Evolution of solitons whose relative amplitude changes from $r=0.8$ to $r=2.5$, the separation distance

$$
\mathrm{q} 0=3.5 \text { the relative phase } \theta=3 \pi / 2
$$

\subsection{Evolution of Solitons from Perturbation}

In realistic communication system, the soliton propagation is a ected by many perturbed related to the input pulse shape, chirp and power, amplifier noise, optical filter, modulator, and son. The perturbed NLSE as the presence of small fiber loses is given by

$$
\begin{aligned}
i \frac{\partial \mu}{\partial \xi}+\frac{1}{2} \frac{\partial^{2} \mu}{\partial \tau^{2}}+|\mu|^{2} \mu & =i \xi(\mu) \\
& =-\frac{i}{2} \Gamma \mu
\end{aligned}
$$

The solution of the perturbed NLSE can be written as $[10,13]$

$$
\mu(\xi, \tau)=\eta(\xi) \operatorname{sech}[\eta(\xi)(\tau-q(\xi))] \exp [i \phi(\xi)-\delta(\xi) \tau]
$$

Where

$$
\begin{aligned}
& \Gamma=\alpha L_{D}=\alpha T_{0}^{2} /\left|\beta_{2}\right| \\
& \eta(\xi)=\exp (-\Gamma \xi) \\
& \phi(\xi)=\phi(0)+[1-\exp (-2 \Gamma \xi)] / 4 \Gamma
\end{aligned}
$$

It has been assumed that $\eta(0)=1, \delta(0)=q(0)=0$. Both $\delta$ and q remain zero along the fiber. As it is observed on fig.6, the solitons propagation in the lossy fiber at the presence of GVD and SPM turns out to be remain stable and survived except a quasi reduction in the intensity of the envelope simulated over $500 \mathrm{~km}$.

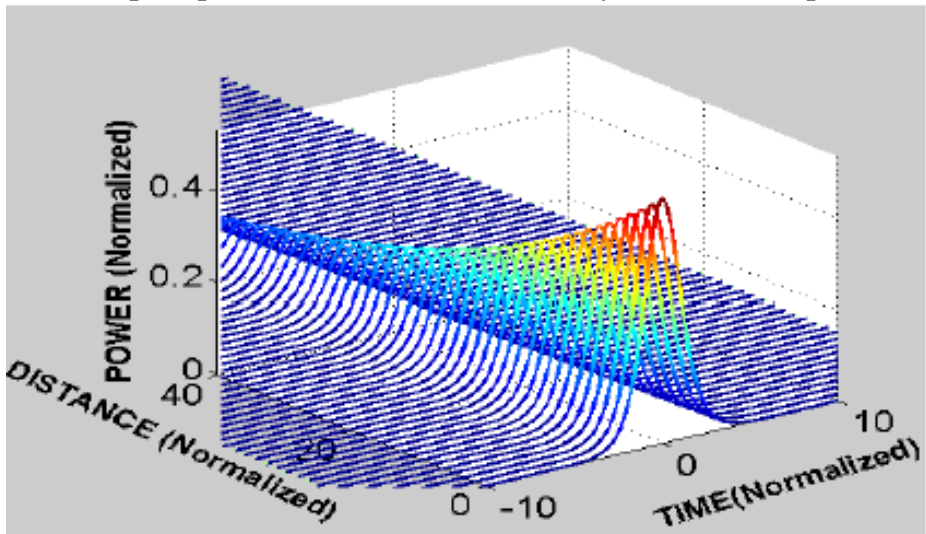

Figure 6: The evolution of solitons in the presence of small perturbation 


\subsection{Evolution and Interaction of Dark Solitons in kerr medium}

The name dark soliton is emerged as the intensity profile associated with dark solitons exhibiting a dip in a uniform background. By assuming the general solution of eq.(6),the following function is considered as solution called fundamental dark soliton[6,11, 13, 14]

$\mu(\varepsilon, \tau)=r\left\lfloor B \tanh (\varepsilon)-i \sqrt{1-B^{2}}\right\rfloor \exp (i \eta \xi)$

Where $\xi=\eta B \tau-\tau_{s}-\eta B \sqrt{1-B^{2}}$. The parameter $\eta$ and $\tau_{s}$ represent the black ground and dip location respectively. B governs the depth of of the $\operatorname{dip}(|\mathrm{B}| \leq 1)$. For $\mathrm{B}=1$, the intensity at the dip center falls to zero. Dark solitons exhibit several interesting features. Consider a black soliton whose canonical form, obtained from eq.(15), after choosing $r=1$ and $B=1$, is given by

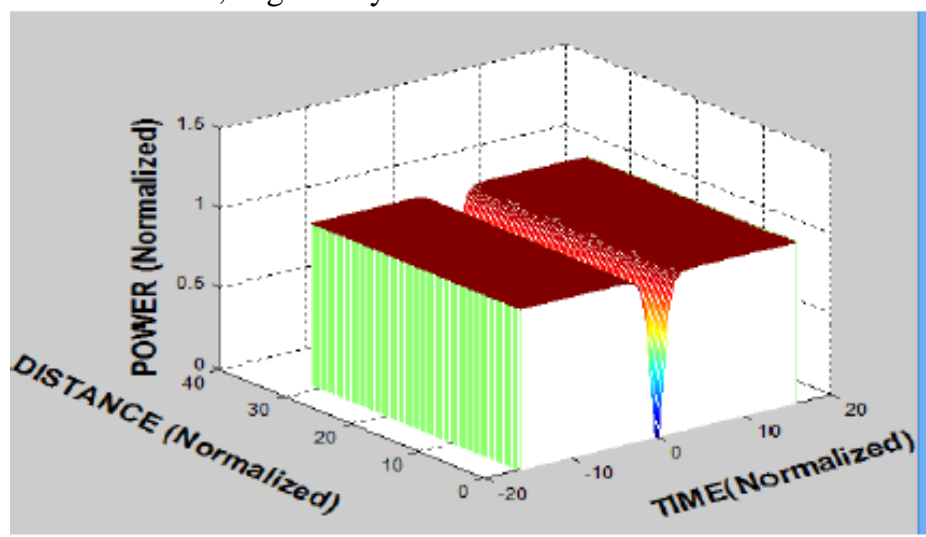

Figure 7: The power distribution of dark soliton for several values of internal phase. The intensity drops zero at the center for black solitons .

$\mu(\varepsilon, \tau)=\tanh (\tau) \exp (i \eta \xi)$

The numerical simulation of eq(16) with the help of SSFM algorithm by solving eq(6) would indicate the distribution of intensity with relatively broad and intensity dropped in the middle shown on fig.7. Experimental realization of dark soliton is possible only with a finite background instead of infinite background associated with the ideal dark solitons[13]. In practice, a pulse with a narrow dip at its center is used to excite a dark soliton.

\subsection{Interaction of two dark Solitons}

A system of coupled nonlinear Schrodinger equations has dark soliton solutions in nonlinear Kerr medium. The interaction of two dark soliton with the same or different phase and amplitude generates new electromagnetic envelopes that are quite different in pulse or soliton in appearance from the fundamental dark soliton when it is studied in nonlinear dispersive medium or Kerr medium [15]. For the simulation of interaction of two dark solitons, the superimpose of the following two dark solitons have been taken in the following form as the solution of coupled nonlinear Schrödinger equations (CNLSEs) described as [16, 17]

$\mu_{\text {sum }}=r\left(\tanh \left(r\left(\tau+q_{0}\right)-i\right) e^{i\left[r\left(\tau+q_{0}\right)-5 r^{2} \xi\right]}+r\left(\tanh \left(r\left(\tau-q_{0}\right)\right)-i\right) e^{i\left[r\left(\tau-q_{0}\right)-5 r^{2} \xi\right]}\right.$

Where $\mathrm{q}_{0}$ is the distance from one dark soliton to the origin and $\mathrm{r}$ is the relative amplitude of the soliton. For easier simulation, it is available to take the modulus or the absolute value of eq.(17) to be conveniently simulated by SSFM algorithm.

$$
\begin{aligned}
& \left|\mu_{\text {sum }}\right|=r\left[\left[\tanh ^{2}\left(r\left(\tau+q_{0}\right)\right)+\tanh ^{2}\left(r\left(\tau-q_{0}\right)\right)+\right.\right. \\
& \left.\tanh \left(r\left(\tau+\tau_{0}\right)\right) \tanh \left(r\left(\tau-q_{0}\right)\right)\right] \cos \left(2 r q_{0}\right)-2 \tanh \left(r\left(\tau+\tau_{0}\right)\right) \sin \left(2 r \tau_{0}\right) \\
& \left.+2 \tanh \left(r\left(\tau-q_{0}\right) \sin \left(2 r q_{0}\right)\right)+2 \cos \left(2 r q_{0}\right)\right]^{\frac{1}{2}}
\end{aligned}
$$




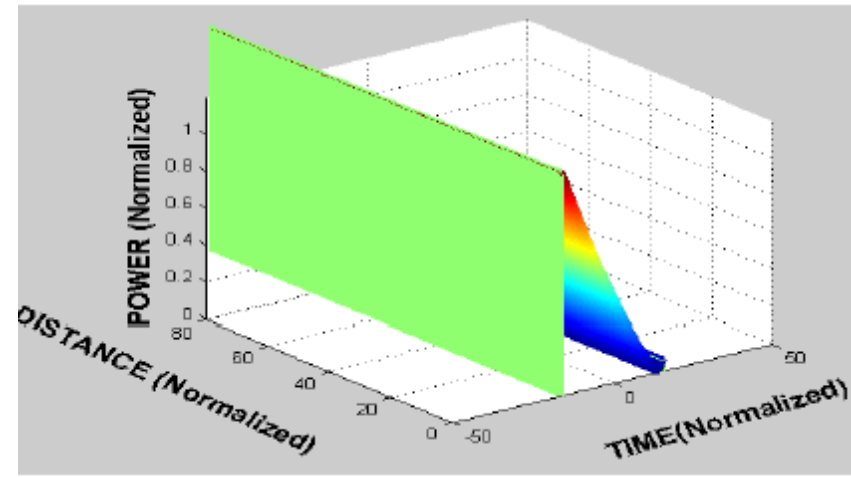

Figure 8: This figure shows the interaction of the two dark solitons and the generation of stable bright electromagnetic pulse. The degree of symmetry distribution of the envelope along the propagating direction are adjusted by $r=0.01, q_{0}=6000$.

The dimensionless numerical values that affecting symmetric distribution of the t evolved envelope of solitons are $r$ and $q_{0}$ along $\mathrm{Z}$ direction. The amplitude ratio for this particular setup $r=0.01$, and the periodic separation of the the two dark solitons from the origin $\mathrm{q}_{0}=6000$ beyond the range of collision are chosen on the discussion under section 3.2. The number of iteration for running simulation over iterated segment $\mathrm{h}=0.9$ is 10,000. From this simulation result of fig.8, the evolved solitons by the superimposed of the two dark solitons are emerged as unfamiliar symmetric 'bright' envelope whose distribution spearing out finitely. Moreover the stability of the emerged 'bright' solitons from interaction of two dark solitons in the presence of perturbation or attenuation parameter $\alpha$ has little effect as demonstrated on fig.9. But there is a gradual reduction of the intensity of the envelope as propagated along the medium.

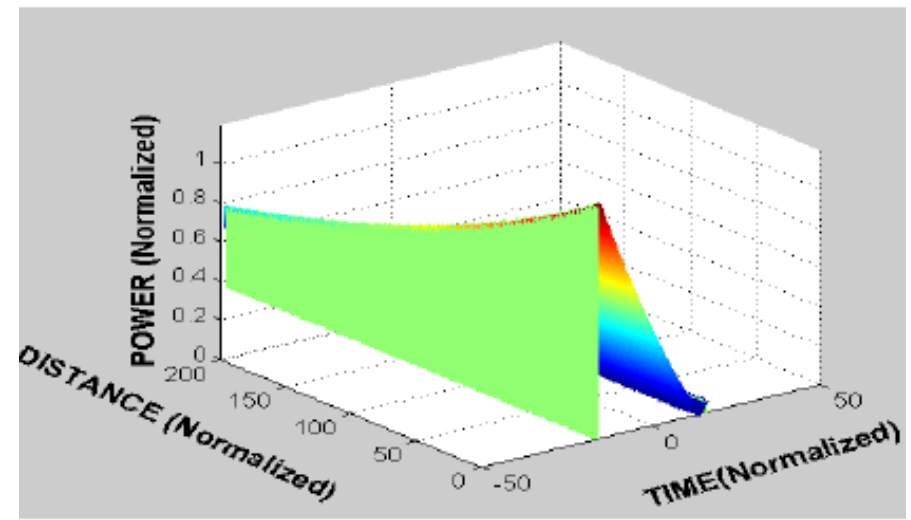

Figure 9: This figure shows the emergence of the 'bright' solitons from the superimpose of two dark solitons in the lossy fiber. The degree of symmetry distribution of the envelope along the propagating direction are adjusted by $\mathrm{r}=0.01, \mathrm{q}_{0}=6000$.

\section{Conclusion}

The study of solitons has received a great deal of attention in recent years both as topics in mathematical physics and because of interest in application to nonlinear and optical communication. Bright solitons have been extensively studied theoretically and their dynamical properties have been verified in numerous experiments over the past decades. On the other hand, dark solitons are less well understood both theoretically and experimentally. According to this paper, long range optical communication involving interaction of dark solitons in the fiber is more efficient than the conventional fundamental (bright) soliton by the following findings.

* Interaction of dark solitons can coupe up perturbation, temporal changes and frequency chirping from unbalanced impact of SPM and GVD.

* The distribution of the evolved solitons is finitely spread out. The likely occurrence of inter symbol interference is lesser than that of the fundamental soliton. Since bright fundamental soliton is Gaussian in nature and its evolved solitons of the envelope spread out spatially in the space. 


\section{References}

[1]. \{Agrawal, Govind P ,Fiber-Optic Communication Systems (John Wiley,2002, p404-415).

[2]. F.S.Ferreira, ,Nonlinear Effects in Optical Fibers (John Wiley, p155-192)

[3]. Gerd Keiser, Optical Fiber ommunications, (McGraw-Hill, 2011, p482-488)

[4]. Taha, Salah Al-Deen Adnan and Shellal, Mehdi M and Kadhim, Ahmed Chyad, Simulation of Gaussian Pulses Propagation Through Single Mode Optical Fiber Using MATLAB, Iraqi Journal of Science, 2013, Vol.54, No.3, p.601-606

[5]. Govind P.Agrawl,Nonlinear Fiber Optics4th edition, (Elsevier,2007,26-112)

[6]. Fedor Mitscheke, Fiber Optics physics and Technology ( Springer 2009,p153-178)

[7]. Ajoy Ghatak, K. Thyagarajan,,Introduction to Fiber Optics (Cambridge University 1998,339-359)

[8]. Ip, Ezra,Nonlinear compensation using back propagation for polarization-multiplexed transmission, Journal of Lightwave Technology ,v IEEE,olume 28, number 6, p 939-951, 2010,

[9]. Landolsi, Taha,Accuracy of the split-step wavelet method using various wavelet families in simulating optical pulse propagation, Journal of the Franklin Institute Volume 343, no 4, 2006, p458-467,

[10]. Mariof F.S.Ferreira,Nonlinear Eff ects in Optica Fibers (John Wiley \& Sons,2011,p155-215

[11]. T.Schneider,Nonlinear Optics in Telecommunication (Springer,2004,215-236)

[12]. Zhang, Hai-Qiang and Meng, Xiang-Hua and Xu, Tao and Li, Li-Li and Tian, Bo ,2007

[13]. Govind P.Agrawl,Nonlinear Fiber Optics (4th edition,Elsevier,2007,130-160)

[14]. Rongcao Yang,Ruiyu Hao, Lu Li, Zhonghao Li, Guosheng Zhou, Dark soliton solution for higher-order nonlinear Schrodinger equation with variable coefficients ,Optics Communications 242 (2004) 285293 Physical Script, volume75, number 4, p537, yea 2007, IOP Publishing

[15]. Nie, Wenjiang, Optical nonlinearity: phenomena, applications, and materials, Advanced Materials,volume,5, number 7-8, p520545,1993 , 